\title{
Numerical Analysis of Nonlinear Soliton Propagation Phenomena Using the Fuzzy Mesh Analysis Technique
}

\author{
P. Shum and S. F. Yu
}

\begin{abstract}
A novel numerical technique, the fuzzy mesh analysis technique, is developed to study the nonlinear propagation phenomena of solitons in an optical fiber. The main advantage of this technique is the variation of mesh size with the shape of soliton pulses along the propagation distance such that: 1) the calculation efficiency can be enhanced and 2) the number of sampling points can be greatly reduced. It is shown that the fuzzy mesh analysis technique is capable of analyzing the propagation phenomena of high-power solitons, pulse compression, and soliton interaction in an efficient manner.
\end{abstract}

Index Terms-Modeling, optical fiber communication, optical fiber theory, optical solitons.

\section{INTRODUCTION}

$\mathbf{O}$ PTICAL solitons have enormous potential in the application of long-haul high-speed optical fiber communication systems [1], [2]. Soliton phenomena can also be used for signal compression and for switching purposes [3]. It is noted that for short-distance soliton communication (around $1000 \mathrm{~km}$ ), it is possible for the soliton transmission speed to be more than $100 \mathrm{Gbit} / \mathrm{s}$ [4]. However, for long-distance transoceanic soliton communication over $10000 \mathrm{~km}$, the transmission speed is limited to 5-40 Gbit/s due to various dispersion and amplifier conditions [5], [6]. Therefore, the study of soliton propagation in an optical fiber is required in order to optimize the performance of soliton communication systems for various applications.

The inverse scattering method (ISM), perturbation method (PM), split-step Fourier method (SSFM), and Fourier series analysis technique (FSAT) are methods available to study propagation phenomena of solitons in an optical fiber [7]-[13]. ISM gives an analytical solution to the propagation of solitons in a lossless fiber. For higher order solitons, the complexity and difficulty of finding exact solutions using ISM will be greatly increased. If the consideration of fiber loss is required, an exact analytic solution cannot be obtained by using ISM. In order to deal with fiber loss, PM should be adopted. However, the limitation of using PM is that the loss factor in the soliton equation cannot be greater than a certain value, otherwise inaccurate results will be obtained.

SSFM is a numerical technique with which fiber loss can be taken into consideration. However, the disadvantages of

Manuscript received February 12, 1998; revised May 25, 1998. This work was supported by HK-RGC and HKU-CRCG.

The authors are with the Department of Electrical and Electronic Engineering, University of Hong Kong, Hong Kong.

Publisher Item Identifier S 0018-9197(98)07191-7. using SSFM include: 1) a large number of sampling points is required (e.g., larger than 200 sampling points) and 2) fast Fourier transform (FFT) is heavily used to transform solutions between time and frequency domains at each propagation step. It is noted that for a propagation distance of one soliton period FFT can be used as many as 3000 times, and the cumulative errors are unavoidable [12]. In order to minimize the cumulative error, FSAT is developed to operate entirely in the frequency domain. This technique is efficient when the required number of sampling points is small. However, the computational efficiency of FSAT reduces significantly with the increase of sampling points. This is because the number of first-order partial differential equations as well as the computational time increase with the number of sampling points being used in the frequency domain [12], [13].

In this paper, we propose a novel fuzzy mesh analysis technique (FMAT) to minimize the required number of sampling points in solving soliton propagation. In Section II, the soliton equation is solved by splitting the corresponding nonlinear partial differential equation into two simpler parts which can be calculated easily, either analytically or numerically, in the time domain. In addition, the mesh size is controlled by the shape of the soliton pulse such that the number of sampling points used can be minimized. In Section III, the numerical efficiency of FMAT is examined. It can be shown that the FMAT is more efficient than others [7]-[13] in the analysis of higher order solitons, pulse compression, and soliton interaction.

\section{Numerical Method}

\section{A. The Implementation of the Fuzzy Mesh Analysis Technique}

The general nonlinear soliton propagation equation which includes dispersive and nonlinear effects is given by

$$
\frac{\partial u}{\partial x}=j \frac{1}{2} \frac{\partial^{2} u}{\partial T^{2}}+j|u|^{2} u
$$

where $u(x, T)$ is the normalized complex amplitude of the soliton pulse, $x$ is the normalized distance along the direction of propagation, and $T$ is the normalized time. The first and second terms on the right-hand side of (1) are the group velocity dispersion and self-phase modulation, respectively. In order to implement FMAT, the nonlinear expression of (1) is split into two parts, the nonlinear and linear parts which can 


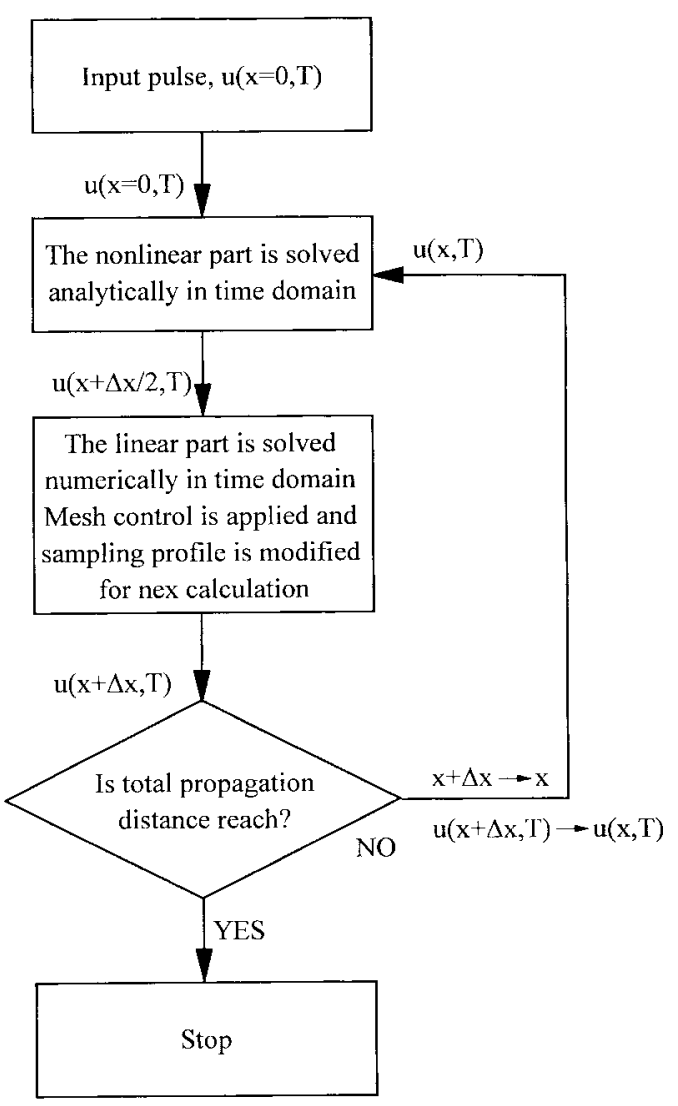

Fig. 1. Flowchart of the FMAT.

be expressed as follows:

$$
\begin{aligned}
& \frac{1}{2} \frac{\partial u}{\partial x}=j|u|^{2} u \\
& \frac{1}{2} \frac{\partial u}{\partial x}=j \frac{1}{2} \frac{\partial^{2} u}{\partial T^{2}} .
\end{aligned}
$$

It is obvious that error that arises from operator splitting is proportional to the choice of $\Delta x$ (i.e., propagation step). This is because as $\Delta x \rightarrow 0, \Delta u / \Delta x \rightarrow \partial u / \partial x$ such that the summation of (2) and (3) gives back to (1). This kind of splitting technique has been applied to discretize the distance variable for the numerical solution of nonlinear partial differential equations. In addition, it is found that the operator splitting in numerical analysis is of second-order accuracy and unconditionally stable [11], [14]-[16]. The nonlinear part (2) can be solved analytically in the time domain, and the linear part (3) can be calculated by finite-element analysis technique.

The use of FMAT in solving (2) and (3) is shown in Fig. 1. The computational procedures can be explained as follows.

1) The input pulse shape $u(x=0, T)$ is set for computation (e.g., $u(x=0, T)=\operatorname{sech}(T)$ for fundamental soliton).

2) The nonlinear part is solved analytically in the time domain for a propagation distance of $\Delta x / 2$.

3) $u(x+\Delta x / 2, T)$ obtained from 2$)$ is used as the initial condition for solving the linear part for another propagation distance of $\Delta x / 2$.

4) Mesh control is adopted each time after the calculation of the linear part such that the sampling profile can be optimized for the next calculation.
5) $u(x+\Delta x, T)$ obtained from 3$)$ is used as the initial condition for solving the nonlinear part of another propagation distance provided that the propagation distance is not reached.

Steps 2)-5) are repeated until the required propagation distance is reached.

In the following paragraphs, the methods to solve the nonlinear and linear parts of (1) as well as the principles of mesh control are discussed in detail.

\section{B. Solution of the Nonlinear Part}

The nonlinear part of (1) can be solved analytically in the time domain. This can be done by solving the first derivative of $|u|^{2}$

$$
\frac{\partial|u|^{2}}{\partial x}=u \frac{\partial u^{*}}{\partial x}+u^{*} \frac{\partial u}{\partial x}
$$

Substituting (2) and its conjugate into (4) with $u(0, T)$ as the initial pulse shape yields an analytical solution of (2) which is given by

$$
u(x, T)=u(0, T) \exp \left[j 2|u(0, T)|^{2} x\right] .
$$

\section{Solution of the Linear Part}

Using the finite difference approximation, (3) can be expressed as

$$
\frac{u_{n+1}-u_{n}}{2 h}=j \frac{1}{2} \frac{\partial^{2} u_{n+1}}{\partial T^{2}}
$$

where $n$ is an integer, $h=\Delta x$, and $u_{n}=u(n h, T)$. Let $M=u_{n}$ and $N=u_{n+1}$, then (6) becomes

$$
N-M=j h \frac{\partial^{2} N}{\partial T^{2}} .
$$

The calculation error of (7) can be estimated from the total residue $R$, defined as

$$
R=\sum_{i=1}^{m} R_{i}
$$

where $i(=1,2,3, \cdots, m)$ is an integer and $m$ is the total number of elements. The residue of the $i$ th element $R_{i}$ is given by

$$
R_{i}=\int_{a_{i}}^{a_{i+1}}\left[\frac{\partial^{2} N}{\partial T^{2}}+j \frac{1}{h}(N-M)\right]^{2} d T
$$

where $a_{i}$ and $a_{i+1}$ denote the node distribution of $u_{n}$.

The solutions of $u$ at the propagation distance, $x$, and $x+\Delta x$ can be expressed as

$$
\begin{aligned}
& M=\sum_{i=1}^{m} M_{i} \\
& N=\sum_{i=1}^{m} N_{i}
\end{aligned}
$$


where $M_{i}$ and $N_{i}$ are defined on the $i$ th element. Using the piecewise cubic interpolant, both $M_{i}$ and $N_{i}$ are given by

$$
\begin{aligned}
M_{i} & =S_{4 i-3} y_{i}+S_{4 i-2} y_{i}^{\prime}+S_{4 i-1} y_{i+1}+S_{4 i} y_{i+1}^{\prime} \\
N_{i} & =S_{4 i-3} z_{i}+S_{4 i-2} z_{i}^{\prime}+S_{4 i-1} z_{i+1}+S_{4 i} z_{i+1}^{\prime}
\end{aligned}
$$

where $y_{i}$ and $y_{i}^{\prime}\left(z_{i}\right.$ and $\left.z_{i}^{\prime}\right)$ are the values of $u_{n}\left(u_{n+1}\right)$ and its gradient, respectively, at the $i$ th node. The parameters $S_{4 i}, S_{4 i-1}, S_{4 i-2}$, and $S_{4 i-3}$ in (12) and (13) are the shape functions of $u_{n}$. In fact, these $S$ parameters can be approximated by general cubic expressions of $T$ which are given as follows:

$$
\begin{aligned}
& S_{4 i-3} \\
& =\frac{a_{i+1}^{2}\left(a_{i+1}-3 a_{i}\right)+6 a_{i} a_{i+1} T-3\left(a_{i}+a_{i+1}\right) T^{2}+2 T^{3}}{\left(a_{i+1}-a_{i}\right)^{3}}
\end{aligned}
$$

$$
\begin{aligned}
& S_{4 i-2} \\
& =\frac{-a_{i} a_{i+1}^{2}+\left(2 a_{i}+a_{i+1}\right) a_{i+1} T-\left(2 a_{i+1}+a_{i}\right) T^{2}+T^{3}}{\left(a_{i+1}-a_{i}\right)^{2}}
\end{aligned}
$$

$$
\begin{aligned}
& S_{4 i-1} \\
& =\frac{a_{i}^{2}\left(3 a_{i+1}-a_{i}\right)-6 a_{i} a_{i+1} T+3\left(a_{i}+a_{i+1}\right) T^{2}-2 T^{3}}{\left(a_{i+1}-a_{i}\right)^{3}}
\end{aligned}
$$

and

$$
=\frac{-a_{i}^{2} a_{i+1}+a_{i}\left(a_{i}+2 a_{i+1}\right) T-\left(2 a_{i}+a_{i+1}\right) T^{2}+T^{3}}{\left(a_{i+1}-a_{i}\right)^{2}} .
$$

Using (8), the stationary conditions of $\mathbf{R}$ are given as follows:

$$
\frac{\partial R}{\partial z_{j}}=\frac{\partial R}{\partial z_{j}^{\prime}}=0
$$

where $j(=1,2,3 \cdots, m+1)$ is an integer. For the $i$ th element, the stationary conditions

$$
\frac{\partial R}{\partial z_{j}}=\frac{\partial R}{\partial z_{i}^{\prime}}=\frac{\partial R}{\partial z_{i+1}}=\frac{\partial R}{\partial z_{i+1}^{\prime}}=0
$$

must be satisfied. Using (9), the first term of (19) can be expressed as

$$
\begin{aligned}
\frac{\partial R}{\partial z_{i}}= & z_{i} \int_{a_{i}}^{a_{i+1}} F_{4 i-3}^{2} d T+z_{i}^{\prime} \int_{a_{i}}^{a_{i+1}} F_{4 i-3} F_{4 i-2} d T \\
& +z_{i+1} \int_{a_{i}}^{a_{i+1}} F_{4 i-3} F_{4 i-1} d T+z_{i+1}^{\prime} \int_{a_{i}}^{a_{i+1}} \\
& \cdot F_{4 i-3} F_{4 i} d T-\frac{j}{h} \int_{a_{i}}^{a_{i+1}} F_{4 i-3} M d T
\end{aligned}
$$

where

$$
F_{i}=S_{i}^{\prime \prime}+j \frac{1}{h} S_{i}
$$

Other terms of (19) can also be obtained in a similar approach.

With the consideration of all $m$ elements, we can obtain the global system of equations below:

$$
A z=\frac{j}{h} d
$$

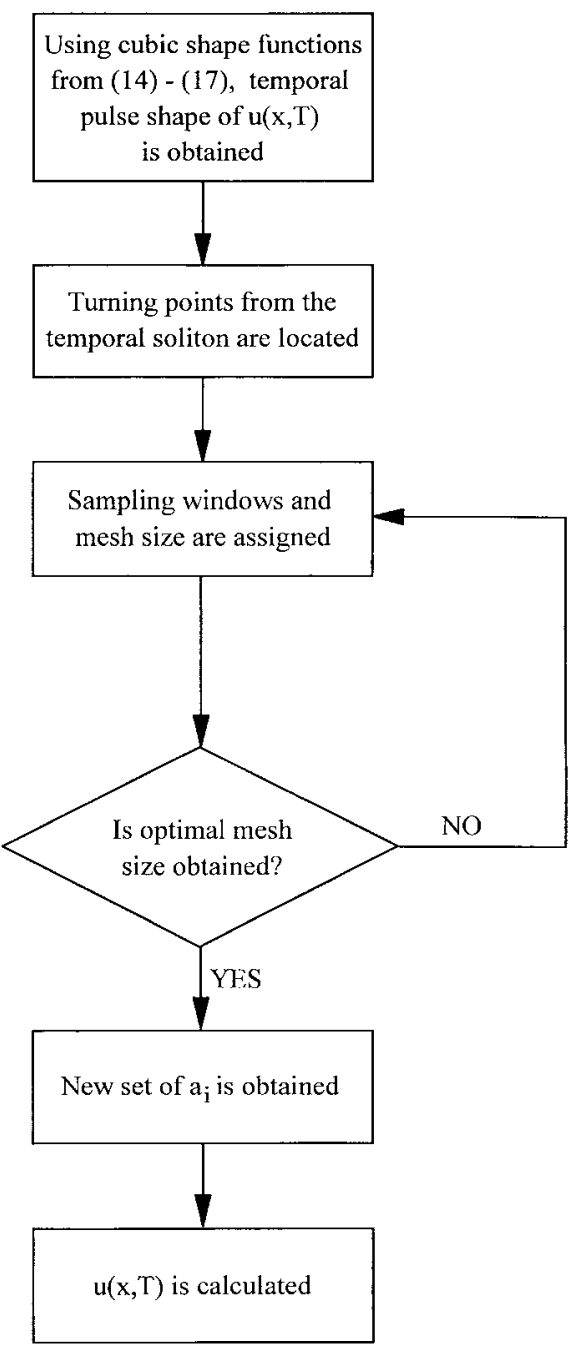

Fig. 2. Flowchart of the mesh control.

where $A$ is defined in (23), shown at the bottom of the next page, and

$$
\begin{gathered}
z=\left[\begin{array}{c}
z_{1} \\
z_{1}^{\prime} \\
z_{2} \\
z_{2}^{\prime} \\
\vdots \\
z_{m+1}^{\prime} \\
z_{m+1}^{\prime}
\end{array}\right] \quad d=\left[\begin{array}{c}
I_{1, M}^{1} \\
I_{2, M}^{1} \\
I_{3, M}^{1}+I_{5, M}^{2} \\
I_{4, M}^{1}+I_{6, M}^{2} \\
I_{7, M}^{2}+I_{9, M}^{3} \\
\vdots \\
I_{4 m, M}^{m}
\end{array}\right] \\
I_{i, j}^{i}=\int_{a_{i}}^{a_{i+1}} F_{i} F_{j} d T
\end{gathered}
$$

and

$$
I_{i, M}^{i}=\int_{a_{i}}^{a_{i+1}} F_{i} M d T .
$$

The above system of equations can be solved to find the values of $z$ and $z^{\prime}$ after a propagation distance of $\Delta x / 2$.

\section{Principle of Mesh Control}

The idea of mesh control is to make use of the available sampling points for minimum calculation error such that the 


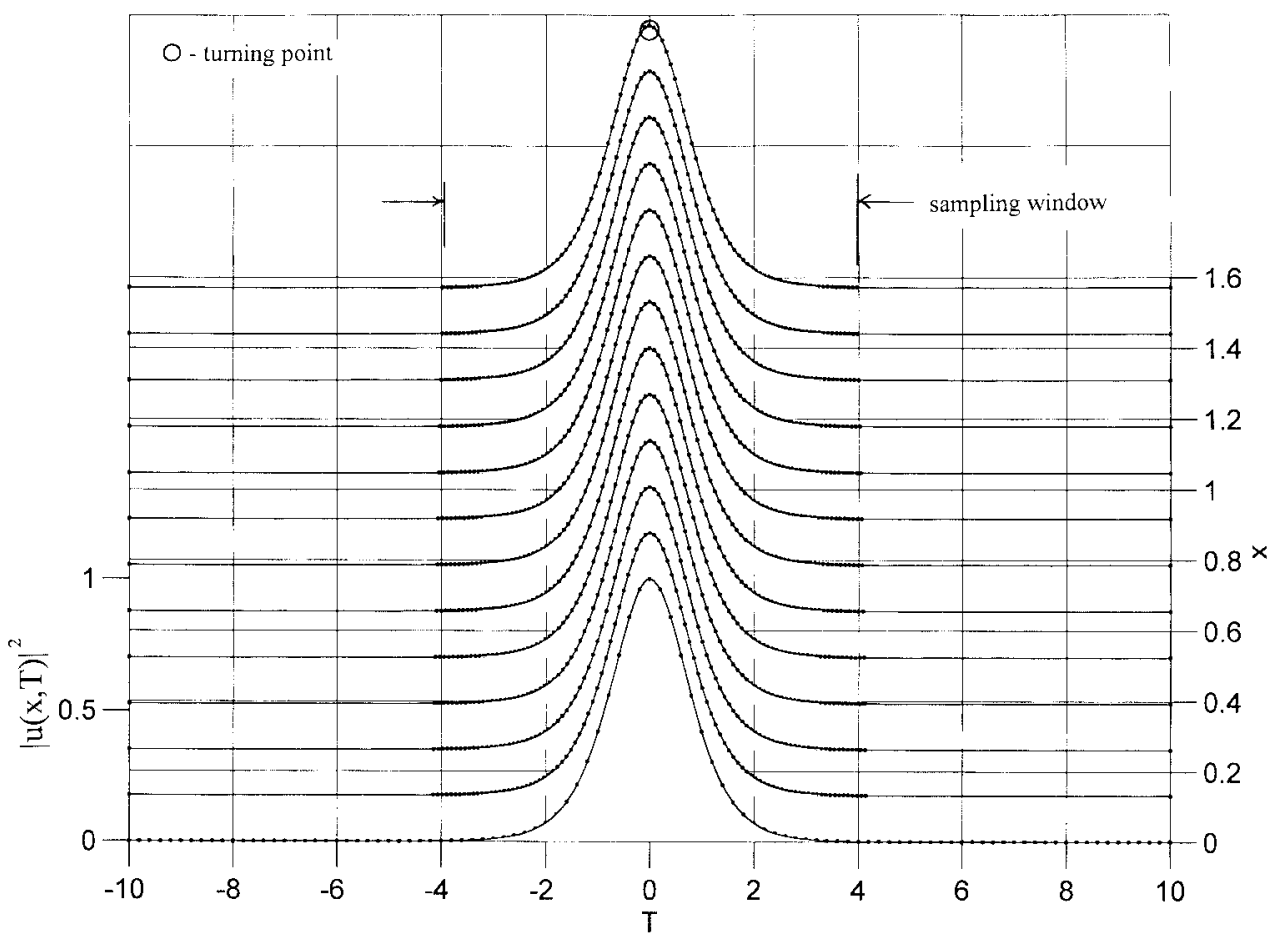

Fig. 3. Propagation of a fundamental nonlinear soliton pulse in an ideal fiber: (a) analytical result obtained by ISM (solid line) and (b) numerical result obtained by the FMAT (solid dots).

numerical model can be more efficient. Different techniques of mesh control such as adaptive grid control have been utilized to solve the linear and nonlinear problems [17]-[21]. The algorithm for the mesh control applied in our analysis is shown in Fig. 2, and the corresponding computational procedures can be explained as follows.

1) Using the cubic shape functions (14)-(17), the temporal soliton pulse shape at a particular propagation distance is deduced as a function of $T$.

2) Based on the calculated cubic shape functions, both the temporal pulse shape and slope of $u(x, T)$ are obtained. Hence, the turning points (i.e., $\partial u(x, T) / \partial T=0$ ) can be located by comparing the variation of the slope of $u(x, T)$.

3) The distribution of sampling points is defined within a sampling window along the $T$ axis. The left and right boundaries of this sampling window are defined as the magnitude of $u(x, T)$ just below $10^{-3}$. Based on the location of turning points, new values of mesh sizes as well as $a_{i}$ are assigned.

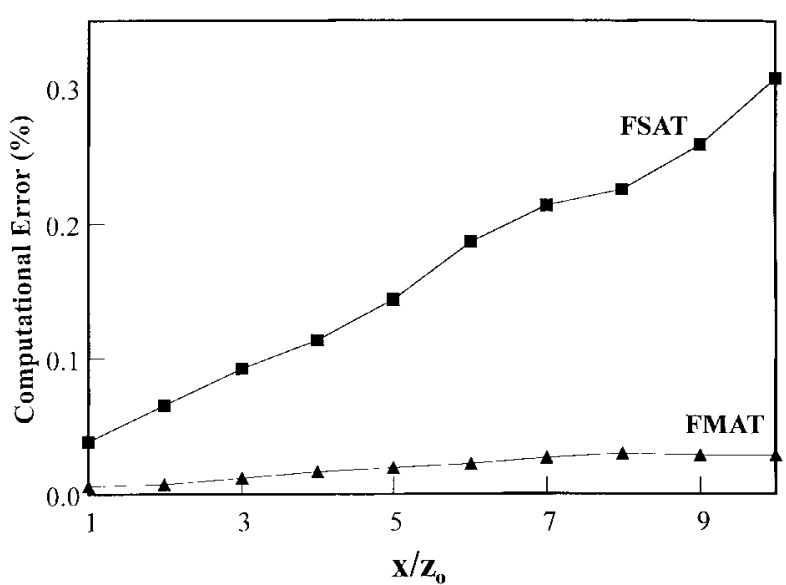

Fig. 4. Computational error versus soliton propagation distance for FSAT and FMAT.

4) The procedures of assignment of mesh sizes can be described as follows.

- The number of turning points $N_{\text {tp }}$ is counted within the sampling window.

$$
A=\left[\begin{array}{ccccccccc}
I_{1,1}^{1} & I_{1,2}^{1} & I_{1,3}^{1} & I_{1,4}^{1} & & & & & \\
I_{1,2}^{1} & I_{2,2}^{1} & I_{2,3}^{1} & I_{2,4}^{1} & & & & \\
I_{1,3}^{1} & I_{2,3}^{1} & I_{3,3}^{1}+I_{5,5}^{2} & I_{3,4}^{1}+I_{5,6}^{2} & I_{5,7}^{2} & I_{5,8}^{2} & & \\
I_{1,4}^{1} & I_{2,4}^{1} & I_{3,4}^{1}+I_{5,6}^{2} & I_{4,4}^{1}+I_{6,6}^{2} & I_{6,7}^{2} & I_{6,8}^{2} & & & \\
& & I_{5,7}^{2} & I_{6,7}^{2} & I_{7,7}^{2}+I_{9,9}^{3} & I_{7,8}^{2}+I_{9,10}^{3} & I_{9,11}^{2}+I_{9,12}^{3} & \\
& & I_{5,8}^{2} & I_{6,8}^{2} & I_{7,8}^{2}+I_{9,10}^{3} & I_{8,8}^{2}+I_{10,10}^{3} & I_{10,11}^{2}+I_{10,12}^{3} & \\
& & & & & \vdots & \vdots & \vdots & \vdots
\end{array}\right]
$$




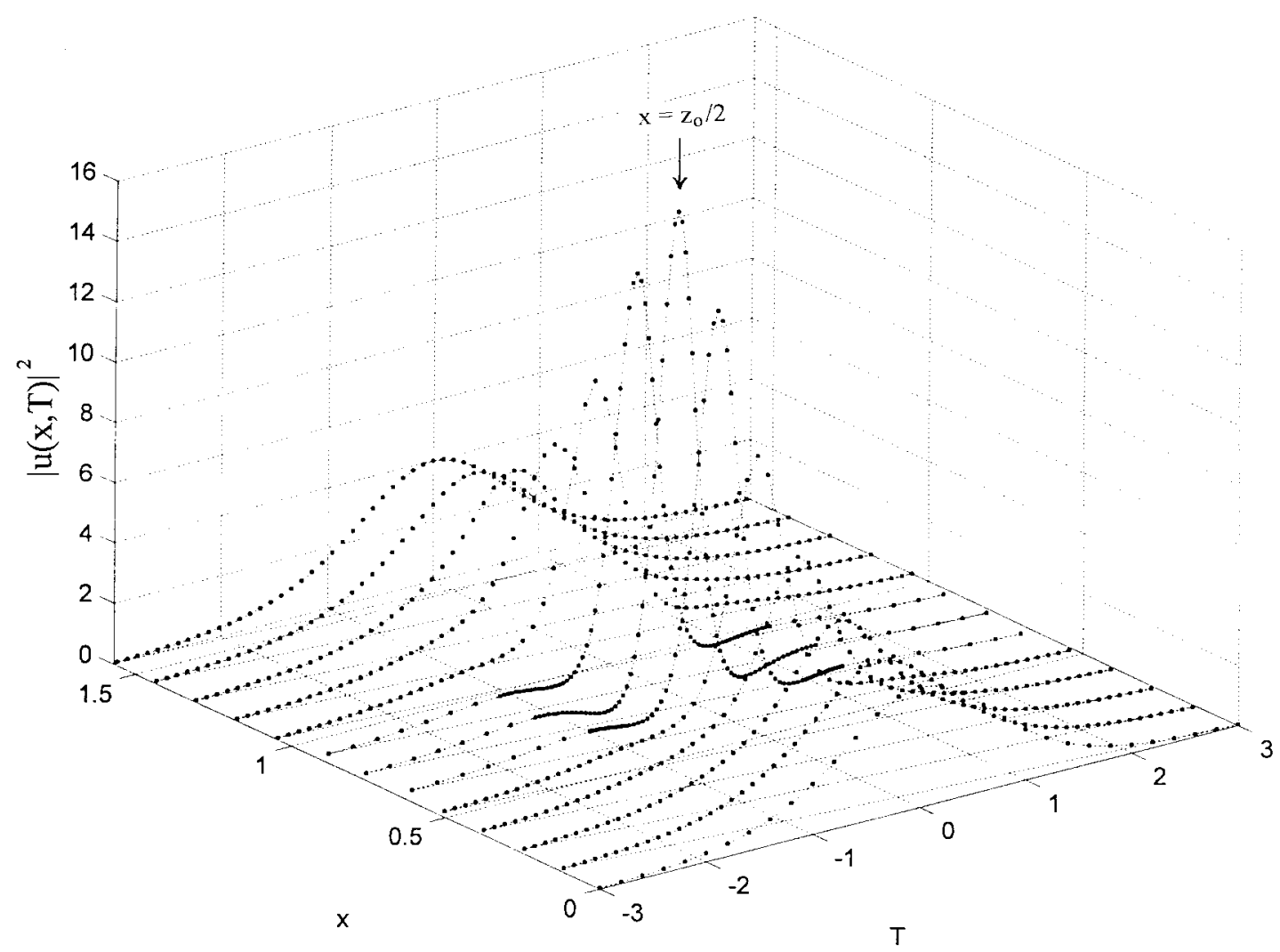

Fig. 5. Propagation of a second-order nonlinear soliton pulse in an ideal fiber. The results (solid dots) are computed by FMAT with mesh control.

- $\quad$ The sampling window is subdivided into $N_{\mathrm{tp}}+1$ regions with the turning points as the boundaries of each subdivided region.

- The sampling points within each subdivided region are equally spaced, but the number of sampling points $N_{j}$ can be different between subdivided regions.

- If $u_{p j}$ and $u_{t j}$ (i.e., $u_{p j}>u_{t j}$ ) are the boundaries of $u(x, T)$ within a subdivided region, $N_{j}$ can be defined as $N_{j}=R_{j} \cdot\left(u_{p j}-u_{t j}\right)$, where $R_{j}$ is a tuning factor. $R_{j}$ is adjusted such that the summation of sampling points within each subdivided region is equal to $N_{t p}$

- If the total number of sampling points assigned to each subdivided region is more (or less) than the available sampling points of the sampling window, the above procedures are repeated for a different $R_{j}$ until the optimal mesh size is achieved.

5) Hence, new values of $u(x, T)$ can be calculated using (13)-(17) with the optimized node distribution $a_{i}$.

Using this mesh control, more information of the soliton pulses can be obtained but without increasing the total number of sampling points. Hence, the computational efficiency and accuracy can be improved with FMAT.

\section{NumericAl RESUltS}

For a fundamental soliton pulse of shape $u(0, T)=$ $\operatorname{sech}(T)$, it is well known that its pulse shape should be unchanged along an ideal fiber. Fig. 3 compares the numerical results (i.e., obtained by FMAT) and exact solutions (i.e., obtained by ISM) for the propagation of a fundamental soliton of one soliton period $z_{o}$ (one soliton period in our presented analysis is equivalent to $\pi / 2$ ). For the numerical calculation, the number of sampling points is set to 100 and one sampling window is utilized. It is observed that the mesh size of the input pulse is evenly distributed but changed dramatically with the propagation distance. In addition, the mesh size is concentrated around the region $-4<T<4$. The numerical calculation obtained by FMAT also shows good agreement with the analytical one. The influence of mesh control in FMAT on the computational speed and accuracy is also analyzed. The calculation given in Fig. 3 is repeated for the case of equidistant spacing (i.e., without mesh control). It is found that the computational time is about the same (approximately $5 \mathrm{~min}$ run on an IBM PC/Pentium with a $200-\mathrm{MHz}$ clock rate), but the calculation error is increased by double when compared with the case with mesh control. Hence, it is shown that the mesh control subroutine only takes up a small amount of CPU time but significantly reduces the calculation error of the numerical model.

In order to demonstrate the computational efficiency of the proposed FMAT, the calculation error of FMAT is compared with that obtained by FSAT. Fig. 4 shows the computational error of FMAT and FSAT for the calculation of long-distance propagation of a fundamental soliton. The number of sampling points used in both techniques is equal to 18 and the computational error is calculated at various propagation distances by comparing with that from exact solutions. The computational 


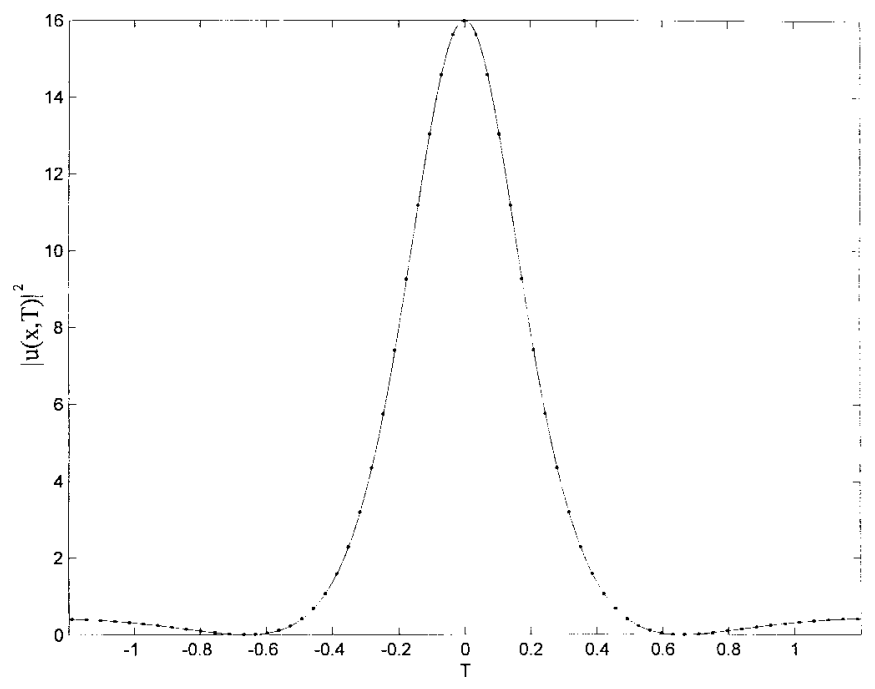

(a)

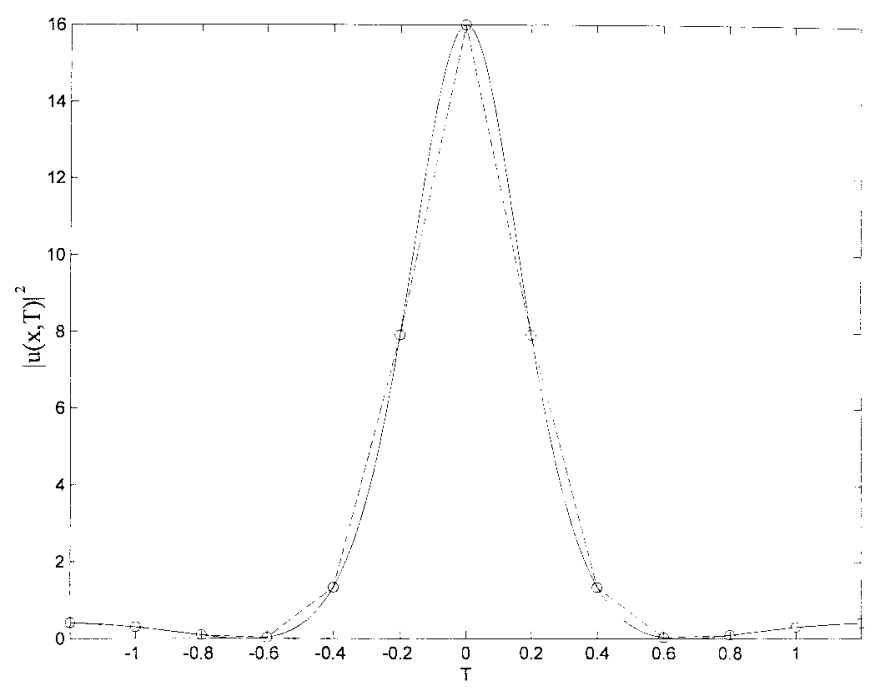

(b)

Fig. 6. Normalized power versus normalized time for a second-order soliton pulse at $x=\pi / 4$ obtained by FMAT (a) with (solid dots) and (b) without (circles) mesh control. The solid line shows the analytical solution of the soliton pulses.

error $\operatorname{Er}(x)$ is defined as

$$
\operatorname{Er}(x)=\sum_{-T_{0}}^{T_{0}}\left|\frac{u_{c}(x, T)-u_{e}(x, T)}{u_{e}(x, T)}\right| \times 100 \%
$$

where $T_{0}=4$ and the subscripts $c$ and $e$ stand for calculated and exact, respectively. As we can see, the computational error arising from FMAT is constant but that by FSAT increases linearly with the propagation distance.

Fig. 5 shows the propagation of a second-order soliton pulse along an ideal fiber for one soliton period. The initial condition

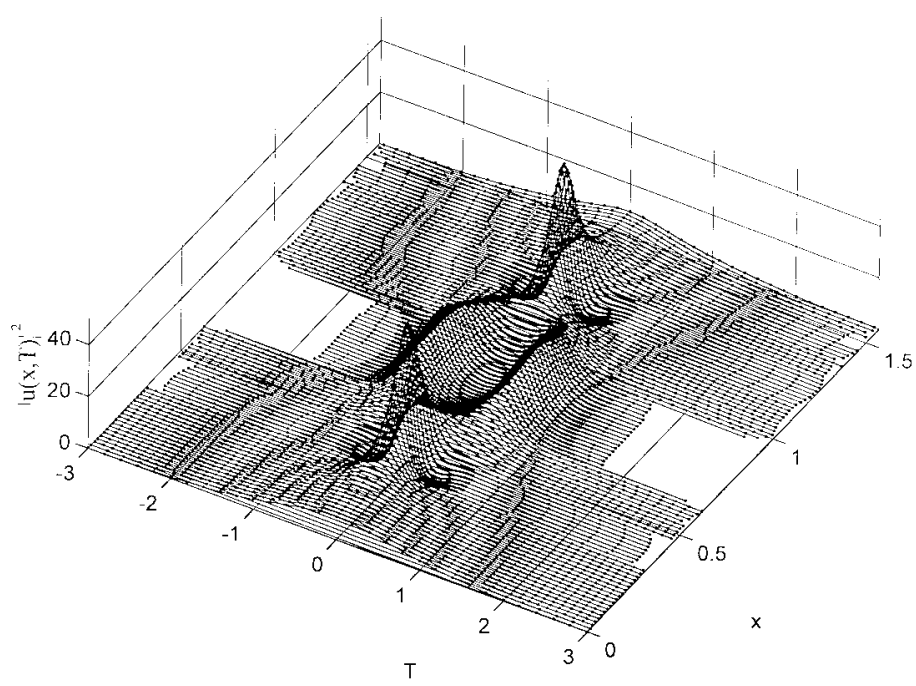

Fig. 7. Propagation of a third-order nonlinear soliton pulse in an ideal fiber. The results (solid dots) are computed by FMAT with mesh control.

of the input soliton pulse is given as $u(0, T)=2 \operatorname{sech}(T)$. In the calculation, the total number of sampling points used is set to 100 (i.e., distributed between $-10<T<10$ ). As shown in the figure, the mesh size and the distribution of the sampling window vary nonuniformly along the propagation distance. It is observed that the sampling points concentrate near the turning points of the soliton pulse. For the propagation distance near $x=z_{0} / 2$ (where the peak power is maximized but the pulsewidth is minimized), the distribution of the sampling window reduces from $-4<T<4$ to $-1<T<1$. The pulse recovers to its original shape at $x=z_{0}$.

For a second-order soliton, the exact solution can be obtained by ISM and is given by (28), shown at the bottom of the page, where $\eta_{1} / \eta_{2}=1 / 3$ for $u(0, T)=2 \operatorname{sech}(T)$. In order to show the computational efficiency of the FMAT, the numerical results of $|u(x, T)|^{2}$ at $x=z_{o} / 2$ computed by FMAT with (solid dots) and without (circles) using mesh control are examined, see Fig. 6 . The solid line shows the analytical solution given by (28). As shown in Fig. 6, the original shape of the soliton pulse is recovered by FMAT with mesh control. However, FMAT without mesh control needs at least five times the number of sampling points to recover the original pulse shape. This is because the rapid change of the soliton pulse shape can be accurately described by the redistribution of mesh size but without increasing the total number of sampling points. It is noted that $63 \%$ of total sampling points are utilized to describe the rapid change of the soliton pulse for the case with mesh control but only $13 \%$ for the case without using mesh control.

Fig. 7 shows the propagation of a third-order soliton pulse along an ideal fiber for one soliton period. The initial condition

$$
u(x, T)=\frac{\frac{4 \eta_{1}\left(\eta_{1}+\eta_{2}\right)}{\left|\eta_{2}-\eta_{1}\right|} e^{-j 2 \eta_{1}^{2} x}\left[\cosh \left(2 \eta_{2} T\right)+\frac{\eta_{2}}{\eta_{1}} \cosh \left(2 \eta_{1} T\right) e^{-2 j\left(\eta_{2}^{2}-\eta_{1}^{2}\right) x}\right]}{\cosh \left[2\left(\eta_{1}+\eta_{2}\right) T\right]+\left(\frac{\eta_{1}+\eta_{2}}{\eta_{2}-\eta_{1}}\right)^{2} \cosh \left[2\left(\eta_{2}-\eta_{1}\right) T+\frac{4 \eta_{1} \eta_{2}}{\left(\eta_{2}-\eta_{1}\right)^{2}} \cos \left[2\left(\eta_{2}^{2}-\eta_{1}^{2}\right) x\right]\right]}
$$


of the input soliton pulse is given by $u(0, T)=3 \operatorname{sech}(T)$. The number of elements used in the calculation is also set to 100 (distributed between $-10<T<10$ ). The solid dots indicate the nonuniform distribution of the sampling points. It is observed that the mesh size as well as the distribution of the sampling window are varied effectively when single or multiple pulses are formed during the propagation. In addition, the original pulse shape of the input pulse is recovered at $x=z_{0}$. This example indicates that FMAT is also capable of controlling the mesh size over high-order pulse compression. The redistribution of sampling points is also possible if more soliton pulses are excited.

\section{CONCLUSION}

Due to the periodic variations of high-power solitons, ultrasharp pulses are being created such that a large sampling density may be required in the numerical calculation. In order to improve the computational efficiency, a new numerical technique, FMAT, is developed. The main advantage of FMAT is the redistribution of mesh size with the shape of soliton pulses such that the sampling density can be greatly reduced. It is shown that, in our calculations, with real time control of mesh size taken into consideration, the propagation behavior of high-power solitons can be studied in an efficient manner and the requirement of the total number of sampling points is drastically reduced compared with other existing techniques. Furthermore, FMAT is capable of analyzing high-power soliton propagation, soliton pulse compression, soliton interaction, as well as other nonlinear propagation problems.

\section{REFERENCES}

[1] M. Nakazawa, E. Yoshida, and Y. Kimura, "Ultrastable harmonically and regeneratively modelocked polarization-maintaining erbium fiber ring laser," Electron. Lett., vol. 30, no. 19, pp. 1603-1605, Sept. 1994.

[2] F. Favre, D. Le Guen, M. L. Moulinard, M. Henry, P. L. Francois, F. Devaux, and T. Georges, "Single-wavelength $40 \mathrm{Gbit} / \mathrm{s}, 8 \times 100 \mathrm{~km}$ span soliton transmission without any in-line control," Electron. Lett., vol. 33, no. 5, pp. 407-408, Feb. 1997.

[3] W. S. Man, M. S. Demokan, and H. Tam, "All-optical routing switch using a fiber nonlinear directional coupler and controlling sections," $J$. Lightwave Technol., vol. 14, pp. 2793-2798, Dec. 1996.

[4] M. Nakazawa, E. Yoshida, E. Yamada, K. Suzuki, T. Kitoh, and M. Kawachi, "80 Gbit/s soliton data transmission over $500 \mathrm{~km}$ with unequal amplitude solitons for timing clock extraction," Electron. Lett., vol. 30, no. 21 , pp. $1777-1778$, Oct. 1994.

[5] M. Suzuki, N. Edagawa, H. Taga, H. Tanaka, S. Yamamoto, and S Akiba, "Feasibility demonstration of $20 \mathrm{Gbit} / \mathrm{s}$ single channel soliton transmission over $11500 \mathrm{~km}$ using alternating-amplitude solitons," Electron. Lett., vol. 30, no. 13, pp. 1083-1084, June 1994.

[6] L. F. Mollenauer, E. T. Lichman, M. J. Neubelt, and G. T. Harvey, "Demonstration, using sliding-frequency guiding filters, of error-free soliton transmission over more than $20 \mathrm{Mkm}$ at $10 \mathrm{Gbit} / \mathrm{s}$, single-channel, and over more than $13 \mathrm{Mkm}$ at $20 \mathrm{Gbit} / \mathrm{s}$ in a two channel WDM," Electron. Lett., vol. 29, pp. 910-911, 1993.

[7] C. S. Gardner, J. M. Greene, M. D. Kruskal, and R. M. Miura, "Method for solving the Korteweg-deVries equation," Phys. Rev. Lett., vol. 19, no. 19 , pp. 1095-10976, Nov. 6, 1967.
[8] V. E. Zakharov and A. B. Shabat, "Exact theory of two-dimensional self-focusing and one-dimensional self-modulaton of waves in nonlinear media," Sov. Phys. JETP, vol. 34, no. 1, pp. 62-69, Jan. 1972.

[9] H. A. Haus and M. N. Islam, "Theory of the soliton laser," IEEE J. Quantum Electron., vol. QE-21, pp. 1172-1188, Aug. 1985.

[10] J. A. Giannini and R. I. Joseph, "The propagation of bright and dark solitons in lossy optical fibers," IEEE J. Quantum Electron., vol. 26, pp. 2109-2114, Dec. 1990

[11] R. H. Hardin and F. D. Tappert, "Applications of the split-step fourier method to the numerical solution of nonlinear and variable coefficient wave equations," SIAM Rev., vol. 15, pp. 423-423, 1973.

[12] H. Ghafouri-Shiraz and P. Shum, "A novel method for analysis of soliton propagation in optical fibers," IEEE J. Quantum Electron., vol. 31, pp. 190-200, Jan. 1995.

[13] P. Shum and S. F. Yu, "Improvement of Fourier series analysis technique by time-domain window function," IEEE Photon. Technol. Lett., vol. 8, pp. 1364-1366, Oct. 1996

[14] J. A. C. Weideman and B. M. Herbst, "Split-step method for the solution of the nonlinear Schrodinger equation," SIAM J. Numerical Anal., vol. 23, no. 3, pp. 458-507, June 1986

[15] T. R. Taha and M. J. Ablowitz, "Analytical and numerical aspects of certain nonlinear evolution equations. II. Numerical, nonlinear Schrodinger equation," J. Comput. Phys., vol. 55, pp. 203-230, 1984.

[16] F. If, P. Berg, P. L. Christiansen, and O. Skovgaard, "Split-step spectral method for nonlinear Schrodinger equation with absorbing boundaries," J. Comput. Phys., vol. 72, no. 2, pp. 501-503, Oct. 1987.

[17] Z. Wanxie, X. Zhuang, and J. Zhu, "A self-adaptive time integration algorithm for solving partial differential equations," Appl. Math. Comput., vol. 89, pp. 295-312, 1998.

[18] D. Yevick, J. Yu, and F. Schmidt, "Analytic studies of absorbing and impedance-matched boundary layers," IEEE Photon. Technol. Lett., vol. 9, pp. 73-75, Jan. 1997

[19] X. Xin, H. F. Zhang, and X. X. Zhang, "Semi-adaptive grid method for convection-dominated flow," J. Hydrodynam., vol. 9, no. 1, pp. 96-101, 1997.

[20] K. S. V. Kumar, A. V. R. Babu, K. N. Seetharamu, T. Sundararajan, and P. A. A. Narayana, "Generalized Delaunay triangulation algorithm with adaptive grid size control," Commun. Numerical Methods Eng., vol. 13, no. 12, pp. 941-948, Dec. 1997.

[21] J. Castillo and E. M. Pedersen, "Solution adaptive direct variational grids for fluid flow calculations," J. Comput. Appl. Math., vol. 67, no. 2, pp. 343-370, Mar. 1996

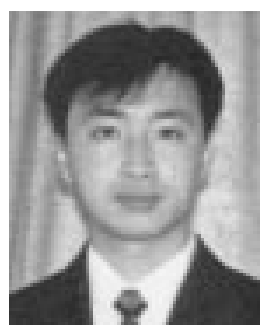

P. Shum was born in China. He received the B.Eng. and Ph.D. degrees in electronic and electrical engineering from the University of Birmingham, U.K., in 1991 and 1995, respectively. His Ph.D. research project involved the analysis of soliton propagation in optical fiber communication systems.

After received the Ph.D. degree, he carried out research in the generation of both optical and electrical solitons in the same university as an Honorary Post-Doctoral Research Fellow. In early 1996, he came back to Hong Kong and carried out research on semiconductor lasers and high-speed optical laser communication in the Department of Electrical and Electronic Engineering, Hong Kong University, as a Visiting Research Fellow. Since July 1997, he joined the Department of Electronic Engineering, Optoelectronics Research Centre, City University of Hong Kong, as a Research Fellow. His research interests are concerned with optical communications, nonlinear waveguide modeling, fiber gratings, and integrated optics.

S. F. Yu, for photograph and biography, see p. 505 of the March 1998 issue of this JOURNAL. 